\title{
Typologie Et Répartition Des Espaces Verts Publics Dans Le Grand Nokoué (Sud Bénin)
}

\section{Adéréwa Aronian Maximenne Amontcha}

Doctorante à l'Ecole Doctorale Pluridisciplinaire (EDP) de l'Université d'Abomey-Calavi (UAC), Bénin

\section{Julien Gaudence Djego}

Enseignant chercheur à la Faculté des Sciences Agronomiques (FSA) de

l'Université d'Abomey-Calavi (UAC), Bénin

\section{Toussaint Olou Lougbegnon}

Enseignant chercheur à l'Ecole de Foresterie et d'Ingénierie de Bois (EFIB-

Kétou) de l'Université Nationale d'Agriculture de Porto-Novo (UNAP), Bénin

Brice Augustin Sinsin

Enseignant chercheur à la Faculté des Sciences Agronomiques (FSA) de

l'Université d'Abomey-Calavi (UAC), Bénin

doi: 10.19044/esj.2017.v13n21p79 URL:http://dx.doi.org/10.19044/esj.2017.v13n21p79

\begin{abstract}
Urban green spaces are essential to ensure the quality of life and the fulfillment of town-dwellers. The objective of this research is to assess the richness of public green spaces in the great Nokoué cities (AbomeyCalavi, Cotonou, Ouidah, Porto-Novo and Sèmè-Podji). The itinerary method was used to find the greens spaces whose list was obtained in the town halls. For each public green space found, the geo-referencing (tracking), the determination of the area of the public places and the measurement of the length of the tracks were made. The results revealed that the great Nokoué cities have 114 public green spaces which can be classified in four types (green Spaces of Tribes 4,39 \%, Green Spaces of Pathways $13,16 \%$, Parks and Squares 26,32 \% and Public Roads Alignment Trees $58,77 \%$ ). Cotonou is the city of the great Nokoué richest in public green spaces $(61,95 \%)$ whereas Ouidah has the highest ratio of public green spaces per inhabitant (Ouidah, $0.27 \mathrm{~m}^{2} / \mathrm{hbt}$, Porto-Novo, $0.18 \mathrm{~m}^{2} / \mathrm{hbt}$, Cotonou, $0.12 \mathrm{~m}^{2} / \mathrm{hbt}$ Abomey-Calavi $0,06 \mathrm{~m}^{2} / \mathrm{hbt}$ and Sèmè -Podji, 0,06 $\mathrm{m}^{2} / \mathrm{hbt}$ ). No city of the great Nokoué has reached the ratio of $10 \mathrm{~m}^{2}$ of public green space per inhabitant as recommended by OMS. It is therefore important that urban authoritys give far greater attention to public green spaces (gardens, parks, squares, etc.) in future development plans.
\end{abstract}


Keywords: Green spaces, great Nokoué, density, ratio of green spaces, georeferencing

\section{Résumé}

Les espaces verts urbains sont essentiels pour assurer la qualité de vie et l'épanouissement des citadins. La présente recherche a pour objectif d'évaluer la richesse en espaces verts publics des villes du grand Nokoué (Abomey-Calavi, Cotonou, Ouidah, Porto-Novo et Sèmè-Podji). La méthode des itinéraires a été utilisée pour retrouver les espaces verts dont la liste a été obtenue dans les mairies. Pour chaque espace vert public retrouvé, le géoréférencement (tracking), la détermination de la superficie des places publiques et la mesure de la longueur des voies ont été faits. Les résultats ont révélé que les villes du grand Nokoué disposent de 114 espaces verts publics qui peuvent être classés en quatre types (Espaces Verts d'Hommages 4,39 \%, Espaces Verts d'Accompagnement de Voies 13,16 \%, Parcs et Squares 26,32 $\%$ et Arbres d'Alignement de Voirie Publique 58,77\%). Cotonou est la ville du grand Nokoué la plus riche en espaces verts publics $(61,40 \%)$ tandis que Ouidah a le ratio d'espaces verts publics par habitant le plus élevé (Ouidah, $0,27 \mathrm{~m}^{2} / \mathrm{hbt}$; Porto-Novo $0,18 \mathrm{~m}^{2} / \mathrm{hbt}$; Cotonou $0,12 \mathrm{~m}^{2} / \mathrm{hbt}$; AbomeyCalavi $0,06 \mathrm{~m}^{2} / \mathrm{hbt}$ et Sèmè-Podji $0,06 \mathrm{~m}^{2} / \mathrm{hbt}$ ). Aucune ville du grand Nokoué n'a atteint le ratio de $10 \mathrm{~m}^{2}$ d'espace vert public par habitant recommandés par l'OMS. Il importe donc que les autorités municipales accordent beaucoup plus d'intérêt aux espaces verts publics (jardins, parcs, squares, etc.) dans les futurs plans de développement.

Mots clés : espaces verts, grand Nokoué, densité, ratio d'espaces verts, géoréférencement.

\section{Introduction}

Les végétaux en villes ont des fonctions écologiques, urbanistiques, sociales et économiques très importants (Lamontagne, 2013). En effet, grâce à la photosynthèse, ils convertissent l'eau et le gaz carbonique en oxygène, contribuant ainsi, à purification de l'air (Gill et al., 2007). Les végétaux possèdent dans leurs tissus des composés fortement antioxydants qui leur permettent de transformer les radicaux libres en molécules non réactives et inoffensives (Brack 2002; Jo 2002; Zhao et al., 2010; Davies et al., 2011; Nowak et al, 2013). Les polluants et poussières en suspension dans l'air se déposent sur les feuillages et sont lavés quand il pleut, limitant ainsi leur circulation dans l'environnement et améliorant la qualité de l'air (Beckett et al., 2000 ; Nowak et al., 2006 ; Janhäll, 2015).

Par ailleurs, les racines des arbres empêchent la dégradation de la structure du sol ou la perte de matériaux soit par ravinement, érosion, 
ruissellement, etc. en maintenant le sol en place dans les terrains en pente (Chen et Jim, 2008 ; Lamontagne, 2013). Leurs feuillages eux, apportent régulièrement de la matière organique pour fabriquer une litière permettant de recouvrir les terrains (Lamontagne, 2013). Les plantes, à travers leurs ombrages, maintiennent des températures beaucoup plus fraîches protégeant ainsi contre la chaleur (Dunnett et Kingsbury, 2004). En effet, ils peuvent baisser la température ambiante de 1 à $5^{\circ} \mathrm{C}$ (Akbari, 2002).

De même, les arbres réduisent la vitesse du vent en offrant une résistance au déplacement de l'air. La vélocité peut être réduite de $50 \%$ sur une distance de 10 à 20 fois la hauteur de l'écran (Lamontagne, 2013). Ils aident aussi à diminuer le bruit ambiant (une ceinture d'arbres de 30 mètres d'épaisseur réduit le bruit de 6 à 8 décibels) (Lamontagne, 2013). En plus, le bruissement des feuilles ajouté au chant des oiseaux qui y nichent, contribue à masquer les bruits de la ville (Nowak et Dwyer, 2000).

Toutes ces utilités du végétal en ville incitent les autorités et certains citadins à les conserver en ville par le biais des espaces verts. En effet, pour que les plantes ne disparaissent pas des villes et que les citadins puissent continuer à bénéficier de ces multiples bienfaits, ces derniers aménagent des jardins fleuris, des espaces plantés d'arbres, des pelouses, etc. dans des cadres privés que publics. C'est le cas dans les villes du grand Nokoué, où, les autorités en charge des villes ont aménagé des espaces verts publics pour i) corriger un tant soit peu, la dégradation du couvert végétal des villes, occasionner par l'étalement urbain, ii) embellir et iii) bénéficier des avantages qu'offrent les plantes en ville. La présente recherche a permis d'évaluer la richesse en espaces verts publics des villes du grand Nokoué et d'analyser leur répartition.

\section{Milieux d'étude}

\section{Situations géographique et administrative des villes du grand Nokoué}

Les villes du grand Nokoué (Porto-Novo, Semè-Podji, Cotonou, Abomey-Calavi et Ouidah) représentent l'espace discontinue constitué par les Arrondissements urbains des Communes du grand Nokoué (les 5 Arrondissements de la Commune de Porto-Novo, les Arrondissements de Sèmè-Podji et d'Agblangandan de la Commune de Sèmè-Podji, les 13 Arrondissements de la Commune de Cotonou, les Arrondissements d'Abomey-Calavi et de Godomey de la Commune d'Abomey-Calavi et les Arrondissements Ouidah I, II, III et IV de la Commune de Ouidah). Elles couvrent une superficie de $380 \mathrm{~km}^{2}$ et sont situées entre $6^{\circ} 18^{\prime}$ et $6^{\circ} 30^{\prime}$ de latitude nord et $2^{\circ} 02^{\prime}$ et $2^{\circ} 40^{\prime}$ de longitude est (figure 1 ). 


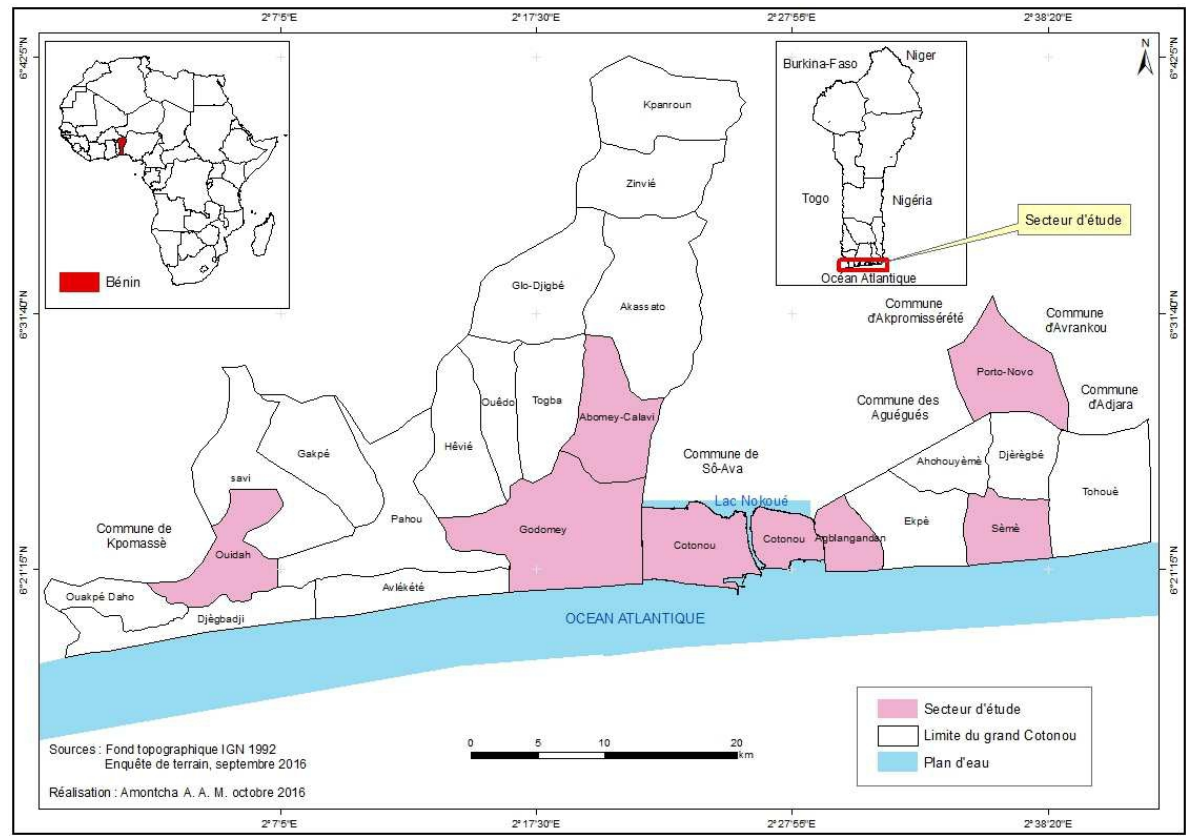

Figure 1 : Situations géographique et administrative des villes du grand Nokoué

\section{Fondements physiques de l'aménagement des espaces verts dans le grand Nokoué}

Le territoire du grand Nokoué est occupé en grande partie de sols hydromorphes, ferralitiques et ferrugineux tropicaux (Blalogoé, 2014). Les sols hydromorphes sont dotés d'une grande capacité de rétention en eau et d'une grande richesse en matières organiques (tourbes) (Lassoudière, 1973). Avec une teneur en potassium $(\mathrm{K})$ moyenne et un taux de phosphore $(\mathrm{P})$ assez élevé (sauf dans les tourbes feuilletées), ils sont à haut potentiel de fertilité (www.fao.org) donc à haut potentiel de développement des espaces verts. Les sols ferralitiques, dotés de bonnes caractéristiques physiques (profondeur, drainage, pénétrabilité), ont par contre, une faible capacité de rétention en eau, un faible taux de potassium $(\mathrm{K})$, de phosphore $(\mathrm{P})$ et de matière organique. Mais avec des épandages périodiques d'engrais et une restitution aux sols des résidus de végétaux ils favorisent le développement des plantes (www.fao.org). Quan aux sols ferrugineux tropicaux, peu profonds et à faible capacité de rétention en eau (Volkoff, 1970, Amontcha, 2013), ils ont une faible teneur en potassium (K), phosphore (P), et en azote (N) (www.fao.org). Mais avec des épandages périodiques d'engrais et une restitution aux sols des résidus de végétaux ils favorisent aussi le développement des plantes. Ce qui constitue un atout pour l'aménagement durable des espaces verts.

Le grand Nokoué est aussi marqué par une situation climatique favorable aux plantes donc à l'aménagement des espaces verts. En effet, il 
est caractérisé par un climat de type subéquatorial humide, avec deux saisons sèches (mi-novembre à mi-mars et mi-juillet à août) et deux saisons pluvieuses (mi-mars à mi-juillet et septembre à mi-novembre). La moyenne pluviométrique annuelle est de $1200 \mathrm{~mm}$, dont 700 à $800 \mathrm{~mm}$ pour la grande saison pluvieuse et 400 à $500 \mathrm{~mm}$ pour la petite saison (Boko, 1988). Les fortes précipitations permettent au sol de retenir une importante quantité d'eau pour répondre aux besoins en eau des plantes. Aussi alimentent-elles les réserves souterraines, plans et cours d'eau des villes grand Nokoué (lac Nokoué $\left(150 \mathrm{~km}^{2}\right)$, lagune de Porto-Novo $\left(35 \mathrm{~km}^{2}\right)$, lagune de Ouidah (40 $\mathrm{km}^{2}$ ), lac Toho (15 $\mathrm{km}^{2}$ ), etc. (Texier, 1980 et Blalogoé, 2014)) qui constituent de grands réserves d'eau pour la satisfaction des besoins hydriques des espaces verts. En effet, l'eau est nécessaire pour la croissance des plantes. Elle permet leur croissance, assure le transport des solutés, les fonctions mécaniques et physiologiques du végétal (maintien du port érigé) et est le support des réactions chimiques internes (Breton et al., 2015).

Fondements humains et sociaux de l'aménagement des espaces verts dans les villes du grand Nokoué

La population du grand Nokoué est passée de 604.106 habitants en 1979 à 1.388 .190 habitants en 2002 pour atteindre 1.984.206 habitants en 2013. Cette région abritera 3.141 .482 habitants en 2025 , soit $25 \%$ de la population béninoise (INSAE, 2013). Cette croissance de la population qui est à l'origine de la forte pression foncière, de l'occupation anarchique des terres et de la grande perte du couvert végétal, accentue la nécessité d'aménager des espaces verts pour non seulement conserver la biodiversité mais aussi, améliorer le cadre et la qualité de vie des populations.

\section{Matériels et méthodes}

\section{Matériel d'étude}

Le matériel d'étude est constitué d'espaces verts publics aménagés dans les villes du grand Nokoué à savoir, les rangers d'ombre (RO) et les places publiques végétalisées (PPV).

\section{Méthodes de collecte des données \\ Critères de choix des espaces verts publics}

Les espaces verts publics pris en compte dans le cadre de cette recherche sont les :

- ranger d'ombre (RO): axes routiers bitumés ou pavés et plantés d'arbre ;

- $\quad$ places publiques végétalisées $(P P V)$ : places publiques sur lesquelles des arbres, des massifs de fleurs, des pelouses sont plantés ; 


\section{Recensement des espaces verts publics dans les villes du grand Nokoué}

La liste des espaces verts publics a été obtenue auprès des mairies. Une fois la liste obtenue, la méthode des itinéraires a été utilisée pour retrouver ces espaces verts sur le terrain et s'assurer qu'ils existent réellement. Pour chaque espace vert public retrouvé, le tracking a été fait, la superficie quand il s'agit d'un PPV et la longueur quand il s'agit d'un RO ont été déterminés à l'aide des fiches de recensement et GPS. Pour les espaces verts dont des obstacles (installation de petits commerces, stationnement de voitures, etc.) empêchent de faire le tour, les coordonnées géographiques de quelques points représentatifs ont étés prises, ce qui a permis de calculer les longueurs et les superficies. Des observations directes ont aussi été faites à l'aide des grilles d'observation et des photos illustratives ont été prises à l'aide d'un appareil photo numérique.

\section{Méthodes de traitement des données et d'analyse des résultats}

L'expression « espace vert» recouvre un large spectre d'espaces. En 1995, l'Association des Ingénieurs des Villes de France (AIVF) a reconnu l'existence de 12 types d'espaces verts (Bouge, 2009) à savoir :

1. Parcs et squares ;

2. Espaces verts d'accompagnement de voies ;

3. Espaces verts d'accompagnement des bâtiments publics (ayant pour rôle la mise en valeur du bâtiment) ;

4. Espaces verts d'accompagnement des établissements industriels et commerciaux ;

5. Espaces verts des établissements sociaux et éducatifs (jardins des crèches, maison de retraite...);

6. Espaces verts des stades et des centres de sport;

7. Cimetières ;

8. Campings, aires d'accueil, villages vacances ;

9. Jardins familiaux ;

10. Etablissements horticoles (serres municipales, lycées horticoles...) ;

11. Espaces naturels aménagés ;

12. Arbres d'alignement à l'unité sur la voirie publique, groupés ou non.

Le classement des espaces verts publics des villes du grand Nokoué a été basé sur cette typologie de l'AIVF. A cette typologie a été complété le type "Espaces verts d'hommage", c'est-à-dire ceux aménagées en mémoire des enfants du Bénin morts pour la paix, communément appelées "Monument aux morts".Après la classification des espaces verts publics des villes du grand Nokoué par type, quelques paramètres ont été calculés :

\section{- Densité (D) en espaces verts des villes du grand Nokoué}

La densité des villes du grand Nokoué en espaces verts publics a été calculée suivant la formule : 


$$
\mathrm{D}_{\mathrm{Evx}}=\frac{n_{E V x}}{S_{V x}} \text { avec : }
$$

$\mathrm{D}_{\mathrm{Evx}}=$ densité des espaces verts dans une ville $\mathrm{x}$;

$\mathrm{n}_{\mathrm{Evx}}=$ nombre d'espace vert dans la ville ;

$\mathrm{S}_{\mathrm{Vx}}=$ superficie total de la ville.

Les résultats obtenus ont été utilisés pour réaliser les cartes de densité en espaces verts des villes du grand Nokoué à l'aide du logiciel ArcGIS.

\section{- $\quad$ Taux de recouvrement}

Pour chaque ville du grand Nokoué, le taux de recouvrement des espaces verts a été calculé suivant la formule :

$$
\mathrm{TR}=\frac{S_{E V x}}{S_{V x}} \times 100 \text { avec : }
$$

$\mathrm{TR}=$ taux de recouvrement des espaces verts dans une ville $\mathrm{x}$; $\mathrm{S}_{\mathrm{EVx}}=$ superficie des places publics végétalisées de la ville; et $\mathrm{S}_{\mathrm{V}_{\mathrm{x}}}=$ superficie total de la ville.

\section{- $\quad$ Ratio espace vert/habitant}

Le ratio espace vert par habitant dans les villes du grand Nokoué a été calculée suivant la formule qui suit :

$$
\mathrm{R}_{\mathrm{EV} / \mathrm{h}}=\frac{S_{E V x}}{E P} \quad \text { avec : }
$$

$\mathrm{R}_{\mathrm{EV} / \mathrm{h}}=$ ratio espace vert par habitant dans une ville $\mathrm{x}$;

$\mathrm{S}_{\mathrm{EVx}}=$ superficie des parcs de la ville ;

$\mathrm{EP}=$ effectif de la population de cette ville.

L'Organisation Mondiale de la Santé (OMS) recommande $10 \mathrm{~m}^{2}$ d'espace vert par habitant :

- $\quad$ si $\mathrm{R}_{\mathrm{EV} / \mathrm{h}}<10 \mathrm{~m}^{2}$ : le ratio espace vert par habitant n'est pas atteint ;

- $\quad$ si $R_{\mathrm{EV} / \mathrm{h}} \geq 10 \mathrm{~m}^{2}$ : le ratio espace vert par habitant est atteint.

Les coordonnées géographiques et les trackings des espaces verts ont été utilisées pour produire des cartes de distribution géographique des espaces verts à l'aide du logiciel ArcGIS.

\section{Résultats}

\section{Densité en espaces verts publics des villes du grand Nokoué}

Les villes du grand Nokoué disposent de 114 espaces verts publics, à raison de 70 dans la ville de Cotonou, 29 dans la ville Porto Novo, 8 dans la ville de Ouidah, 6 dans la ville d'Abomey-Calavi et 1 seul dans la ville de Sèmè-Podji. La figure 2 qui présente la densité en espace verts publics des villes du grand Nokoué illustre mieux cette réalité. 


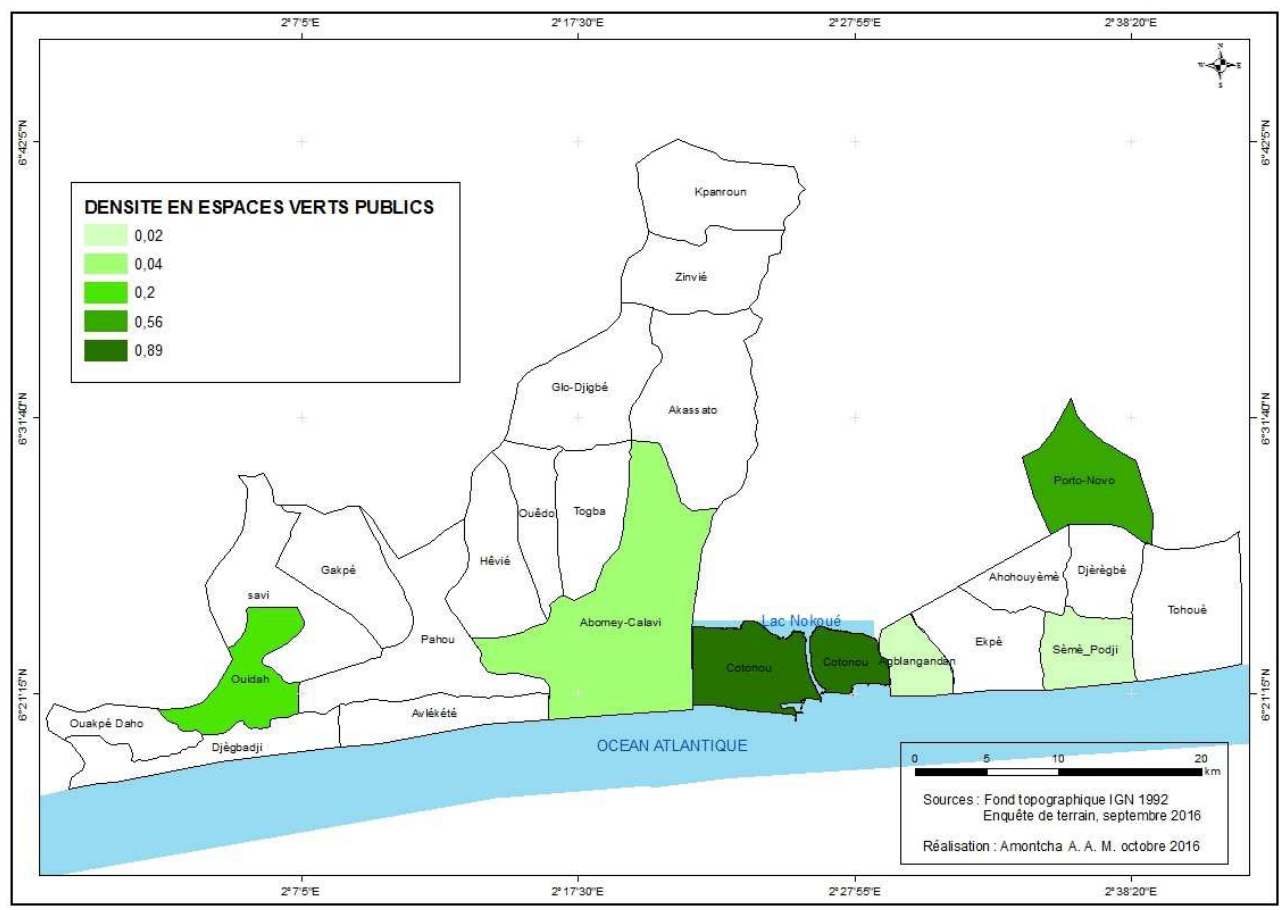

Figure 2 : Densité en espaces verts publics des villes du grand Nokoué

Source : Traitement des données de terrain

Dans le grand Nokoué, la ville de Cotonou est la ville qui a la densité en espaces verts publics la plus élevé $\left(0,89\right.$ espace vert public $/ \mathrm{km}^{2}$ ) (figure 2). Par contre, la ville de Sèmè-Podji la moins dense en espaces verts publics $\left(0,02\right.$ espace vert public $\left./ \mathrm{km}^{2}\right)$. La forte densité en espaces verts publics de la ville de Cotonou peut s'expliquer par le fait que la ville de Cotonou recevait, jusqu'en 2009, environ 350 millions de francs CFA par an et depuis 2010, 10 à 15 millions de francs CFA pour l'aménagement et la gestion des espaces verts publics (Chef du Département des Services de l'Urbanisme et de l'Environnement Urbain (C/DSUEU) de Cotonou). Tandis que les autres villes du grand Nokoué n'ont jamais rien reçu dans ce sens. La faible densité en espaces verts publics de la ville de Sèmè-Podji peut aussi s'expliquer par le fait que les espaces verts ne faisaient pas partie des préoccupations des autorités municipales (Chef Service Technique (CST) de la mairie de SèmèPodji). Selon ce dernier, «la commune n'est pas encore bien urbanisée (il n'y a que les arrondissements de Sèmè-Podji et de Agblangandan qui ont un taux d'urbanisation élevé). C'est maintenant que le problème des espaces verts commence à se poser et nous envisageons lier des partenariats avec les opérateurs économiques pour en aménager ». 


\section{Typologie des espaces verts publics des villes du grand Nokoué}

Les 114 espaces verts publics des villes du grand Nokoué appartiennent à quatre (4) types d'espaces verts à savoir : les espaces verts d'hommages $(\mathrm{EVH})$ (planche 1, d); les arbres d'alignement de voirie publique (AAVP) (planche 1, a); les parcs et squares (PS) (planche 1, b) et les espaces verts d'accompagnement de voies (EVAV) (planche 1, c). La planche 1 illustre les types d'espaces verts publics présents dans le milieu de recherche.
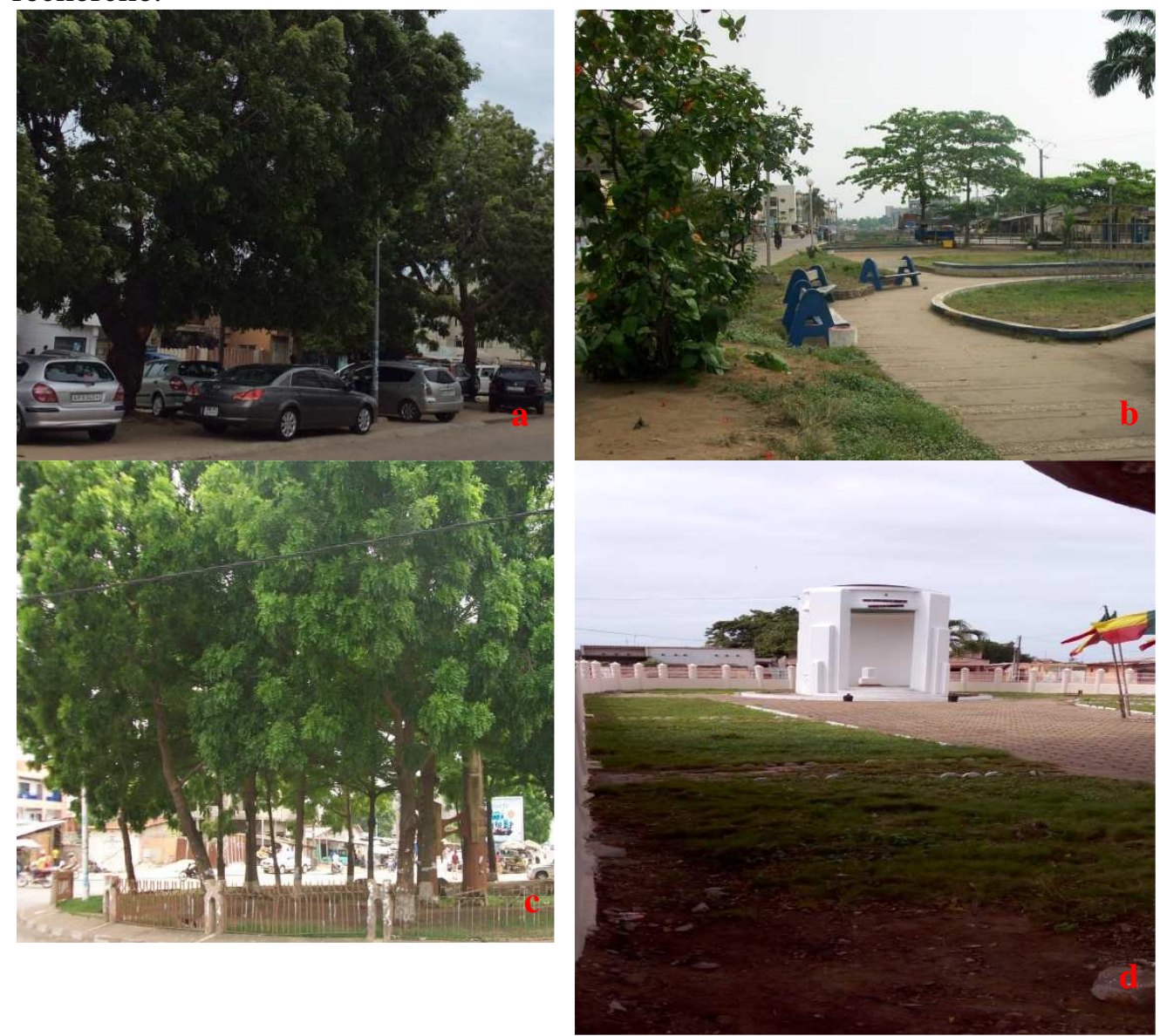

Planche 1 : Images des types d'espaces verts publics des villes du grand Nokoué

Prise de vue : AMONTCHA, Juillet-2016

Le type d'espace vert public le plus dominant dans le grand Nokoué est l'AAVP. En effet, 58,77 \% des espaces verts publics du grand Nokoué sont des AAVP. Seulement 26,32 \% sont des parcs ou squares, lieux par excellence de relaxation et de détente. Les types d'espaces verts sont inégalement répartis dans les villes du grand Nokoué (figure 3). 


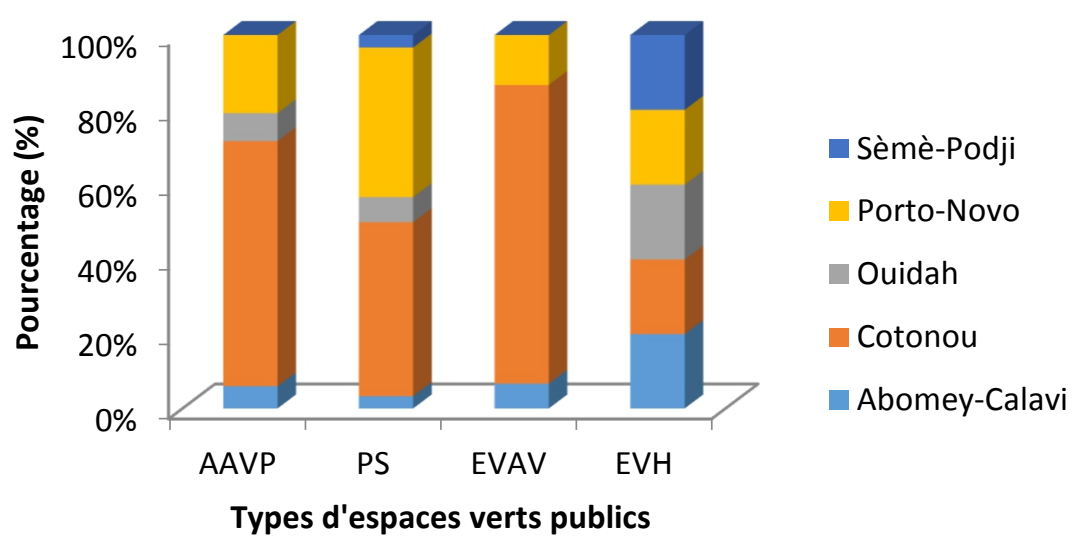

Figure 3 : Répartition des types d'espaces verts publics entre les villes du grand Nokoué Source : travaux de terrain

Quatre-vingt pour cent (80\%) des EVAV, 65,67\% des AAVP et $46,67 \%$ des PS du grand Nokoué sont localisés dans la ville de Cotonou (figure 3) ; $40 \%$ des PS, $20,9 \%$ des AAVP et 13,33 \% des EVAV sont localisés dans la ville de Porto Novo. Seulement 7,46 \% des AAVP du grand Nokoué sont dans la ville de Ouidah et $5,97 \%$ dans la ville d'Abomey-Calavi. Les villes de Ouidah, d'Abomey-Calavi et de Sèmè-Podji regroupent seulement $6,67 \% 3,33 \%$ et $3,33 \%$ des PS. Cotonou est donc la ville la plus riche en espaces verts publics de type EVAV, AAVP et PS. La ville de Sèmè-Podji ne dispose ni d'AAVP, ni d'EVAV. Quant à la ville de Ouidah, elle ne dispose d'aucun EVAV. Toutes les villes du grand Nokoué disposent d'EVH. La figure 4 présente la typologie des espaces verts publics par ville du grand Nokoué.

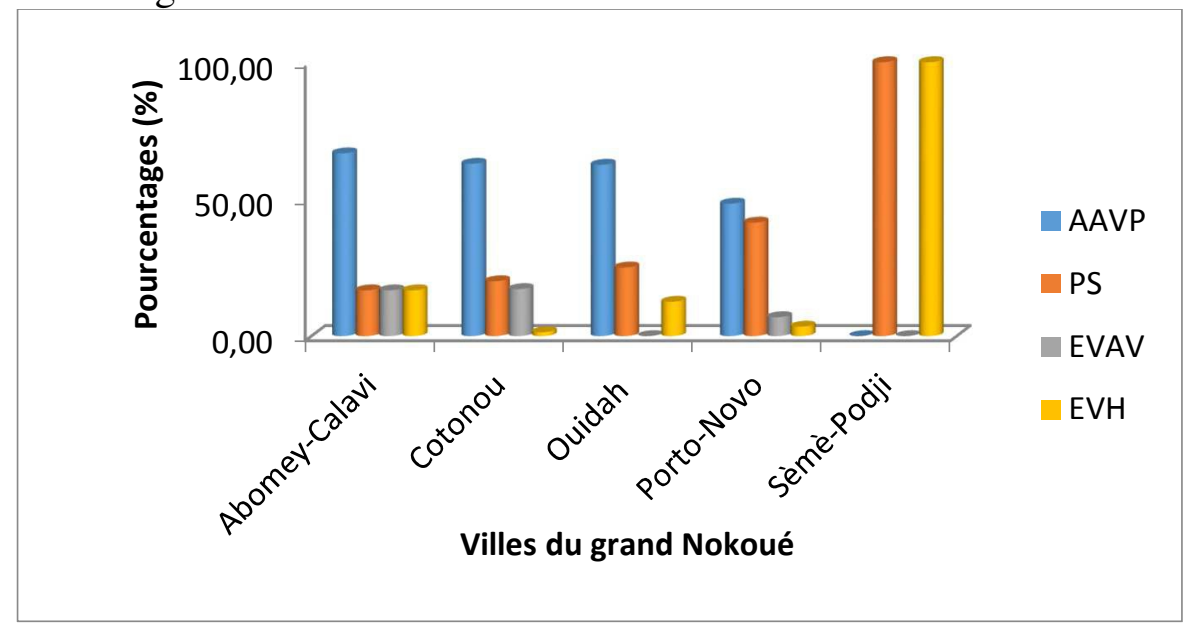

Figure 4 : Typologies des espaces verts publics par ville du grand Nokoué

Source : travaux de terrain 
Les types d'espaces verts publics les plus dominants dans les villes du grand Nokoué sont AAVP (figure 4). En effet, 66,67 \% des espaces verts publics de la ville d'Abomey-Calavi, 62,85\% de Cotonou, 62,5 \% de Ouidah et $48,28 \%$ de Porto-Novo sont des AAVP. Cette situation peut être due au fait que les espaces verts de types AAVP sont moins couteux et demandent moins de place et d'entretien que les autres. Dans la ville de Sèmè-Podji, le seul espace vert public existant est à la fois un EVH et un PS. Cette situation est due au fait que les espaces verts ne faisaient pas partie des préoccupations des autorités de la ville Sèmè-Podji (Cef Services Techniques de la mairie de Sèmè-Podji).

La faible richesse en espaces verts publics des villes du grand Nokoué peut aussi être due au manque d'organisation, d'anticipation et de planification de l'occupation du sol dans le grand Nokoué ; à l'insuffisance de moyens financier et à l'absence de stratégie de recherche de fonds nécessaire à l'aménagement de grand espaces verts.

\section{Taux de recouvrement en places publiques végétalisées des villes du grand Nokoué}

Le taux de recouvrement permet de voir la proportion à laquelle les villes du grand Nokoué sont couvertes par les espaces verts polygonaux. Globalement les villes du grand Nokoué ont une faible couverture en places publiques végétalisées (figure 5).

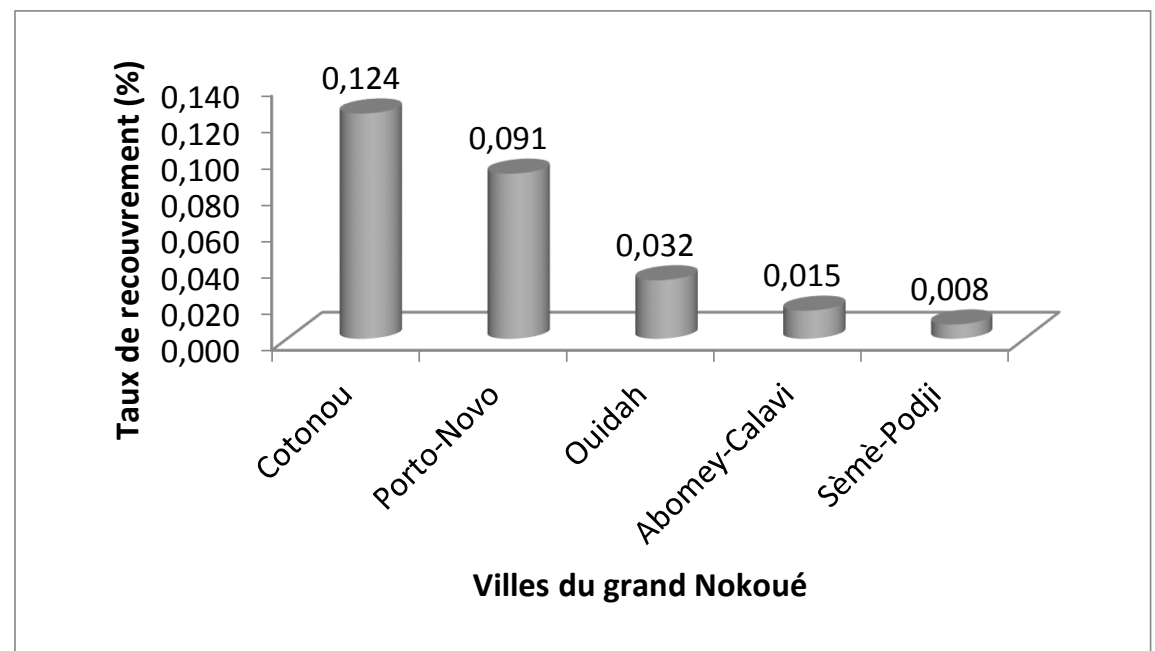

Figure 5 : Taux de recouvrement en place publique végétalisées des villes du grand Nokoué

Source : travaux de terrain

La ville de Cotonou a le recouvrement en place publique végétalisée le plus élevé, avec une couverture de $0,124 \%$ de son territoire (figure 5). La ville de Sèmè-Podji a par contre la couverture en place publique végétalisée 
la plus faible du grand Nokoué avec seulement une couverture de 0,008 \% de sa superficie. Cette situation s'explique par le fait que l'Etat central alloue des fonds à la mairie de Cotonou pour l'aménagement des espaces verts publics, mais pas aux autres villes.

\section{Ratio espace vert par habitant des villes du grand Nokoué}

Le ratio espaces vert par habitant est la quantité d'espaces verts publics disponible pour le loisir et la récréation de chacun des citadins d'une ville. Les villes du grand Nokoué disposent de très peu d'espaces verts publics par habitant (figure 6).

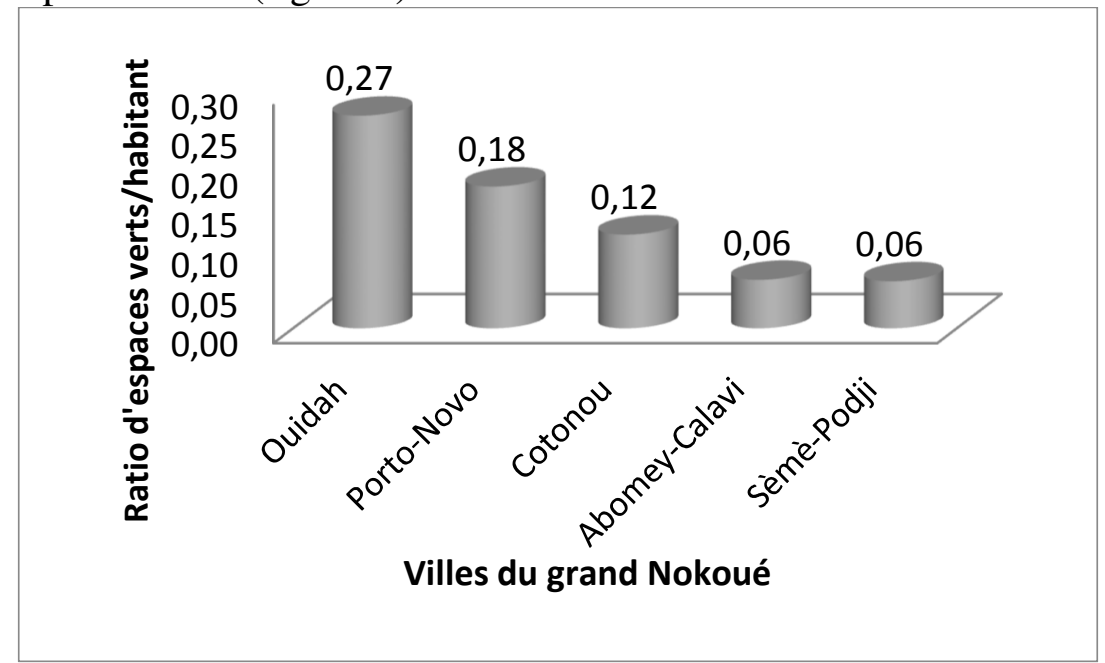

Figure 6 : Ratio espace vert par habitant des villes du grand Nokoué

Source : travaux de terrain

Dans le grand Nokoué, Ouidah est la ville qui a le ratio espace vert par habitant le plus élevé à raison de $0,27 \mathrm{~m}^{2}$ d'espace vert publics par habitant (figure 6). Néanmoins, elle reste très loin de la norme de $10 \mathrm{~m}^{2}$. Abomey-calavi et Sèmè-Podji elles, sont les villes qui ont le ratio espace vert par habitant les plus faible à raison de $0,06 \mathrm{~m}^{2}$ d'espace vert publics par habitant. La faiblesse du ratio d'espaces verts par habitant des villes du grand Nokoué peut s'expliquer par le fait que l'aménagement des espaces verts publics ne fait pas encore partie des priorités des autorités en charge des villes. Par conséquent, ils développent peu de stratégies de mobilisation de ressources (financières, humaines, foncières) pour leur aménagement et leur gestion durable.

\section{Répartition des espaces verts publics des villes du grand Nokoué}

Les espaces verts publics sont inégalement répartis dans les villes du grand Nokoué (figure 7, 8, 9, 10 et 11). 


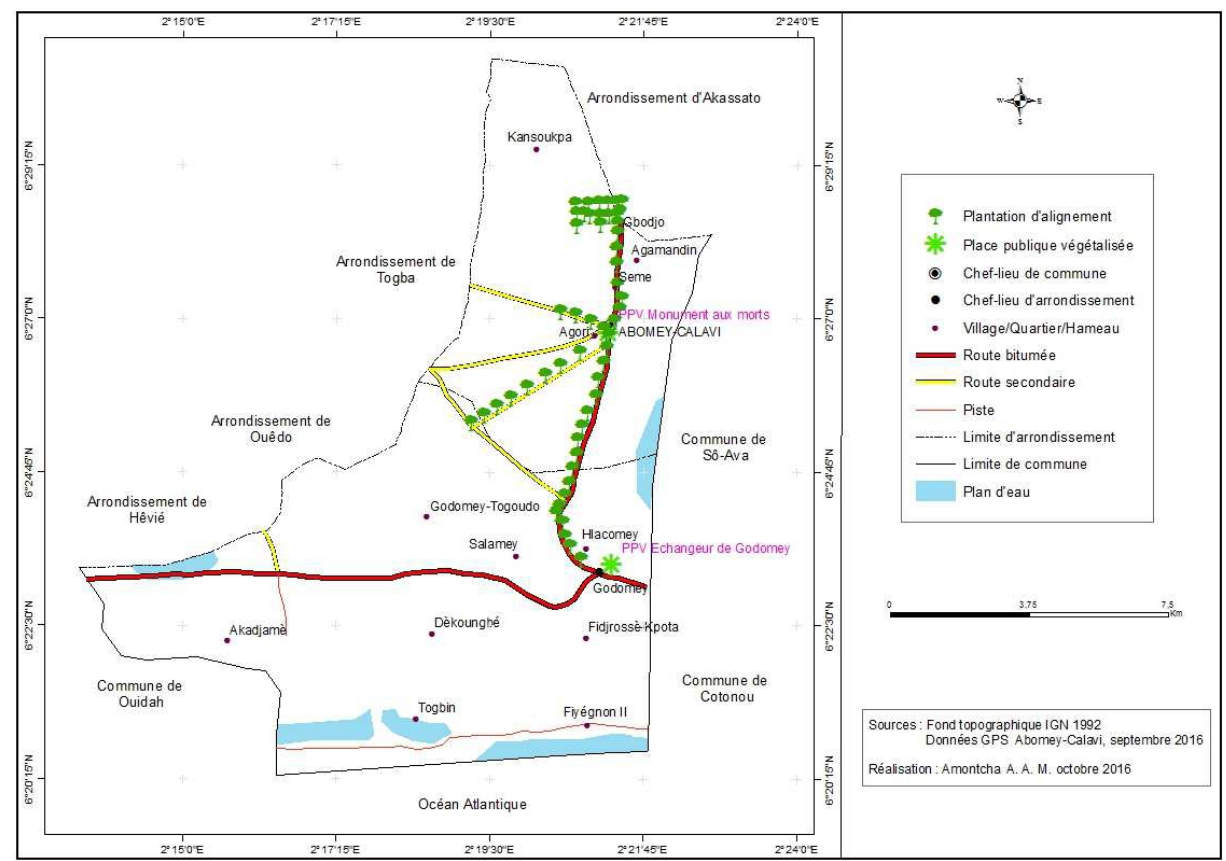

Figure 7 : Répartition géographique des EV Publics de la ville d'Abomey-Calavi

Dans la ville d'Abomey-Calavi, les espaces verts sont concentrés dans l'Arrondissement d'Abomey-Calavi (figure 7). L'Arrondissement de Godomey en dispose très peu.

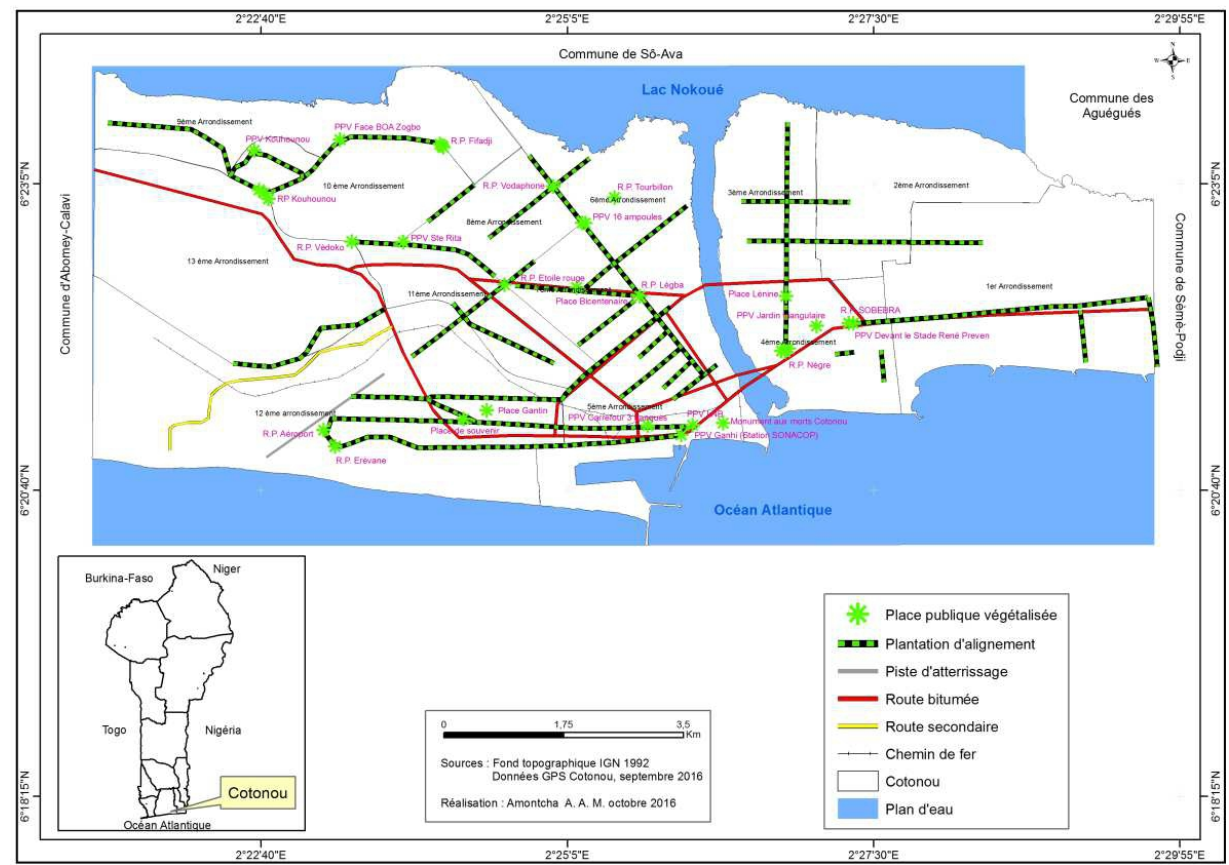

Figure 8 : Répartition géographique des EV publics de la ville de Cotonou 
Dans la ville de Cotonou, les espaces verts publics sont concentrés dans la partie centrale de la ville, plus précisément dans les Arrondissement 4, 5, 7, 8, 11 et 12 (figure 8). Les Arrondissements 2, 9 et 13 sont les moins pourvus.

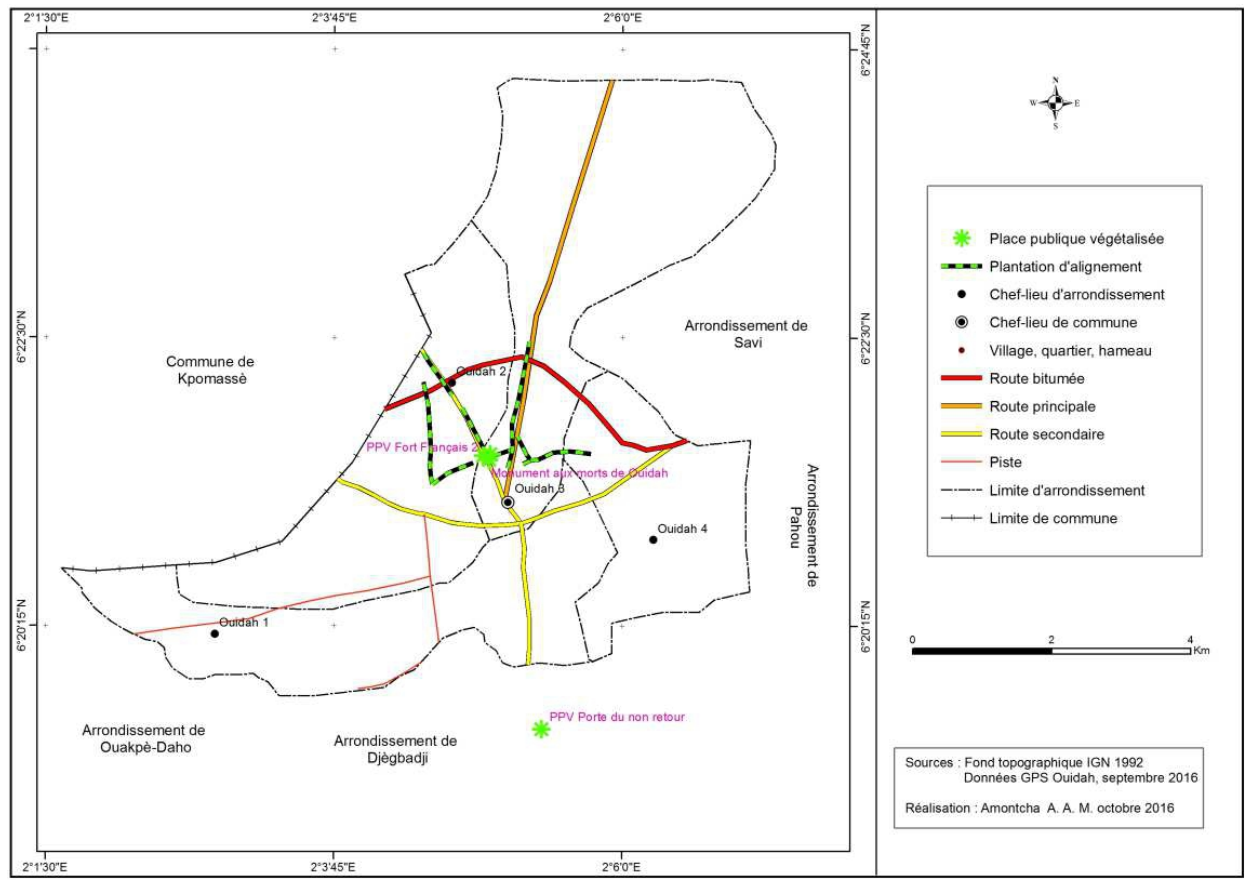

Figure 9 : Répartition géographique des EV publics de la ville de Ouidah

Dans la ville de Ouidah, les espaces verts publics sont concentrés dans les Arrondissement de Ouidah 2 et 3 (figure 9). Une minuscule partie se localise dans l'Arrondissement de Ouidah 4 et celui de Ouidah 1 n'en dispose pas du tout. 


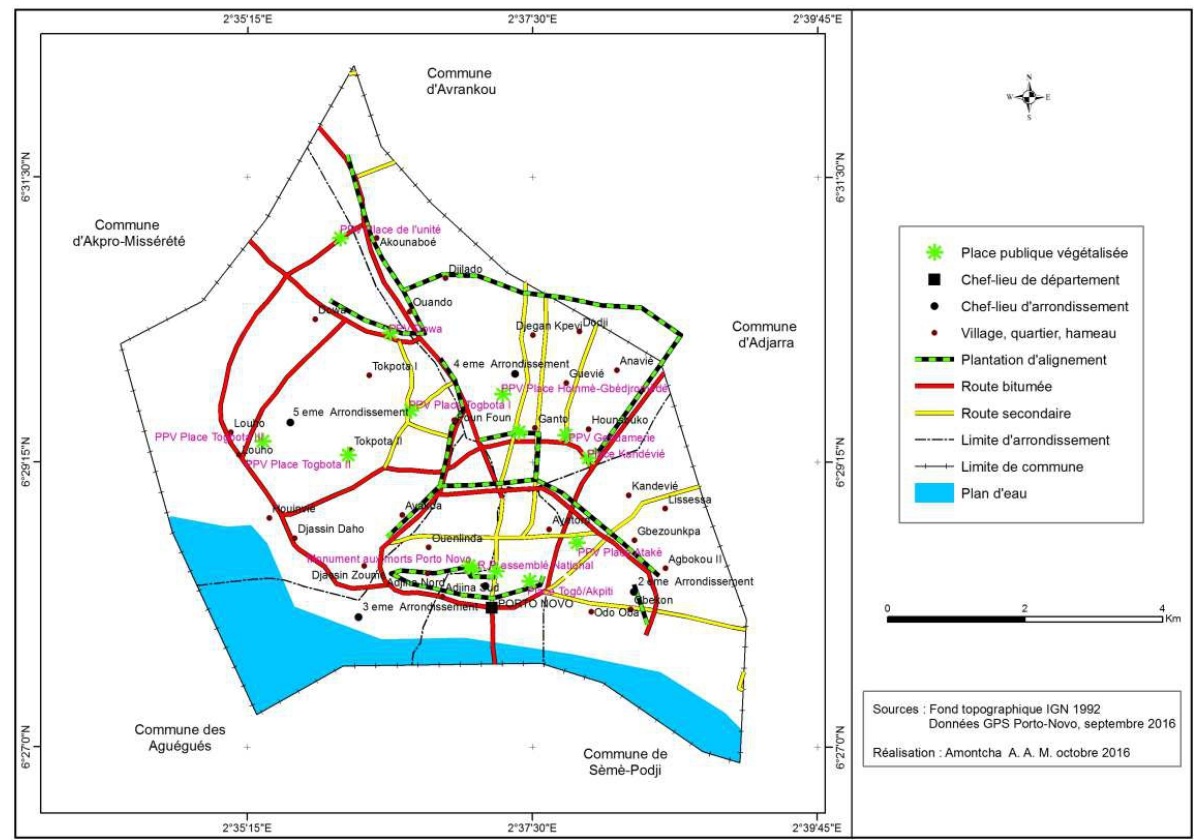

Figure 10 : Répartition géographique des EV publics de la ville de Porto Novo

Dans la ville de Porto-Novo, les $4^{\text {ème }}, 5^{\text {ème }}$ et $1^{\text {er }}$ Arrondissements regroupent le plus grand nombre d'espaces verts publics (figure 10). Les $3^{\text {ème }}$ et $2^{\text {ème }}$ Arrondissements sont peu pourvus.

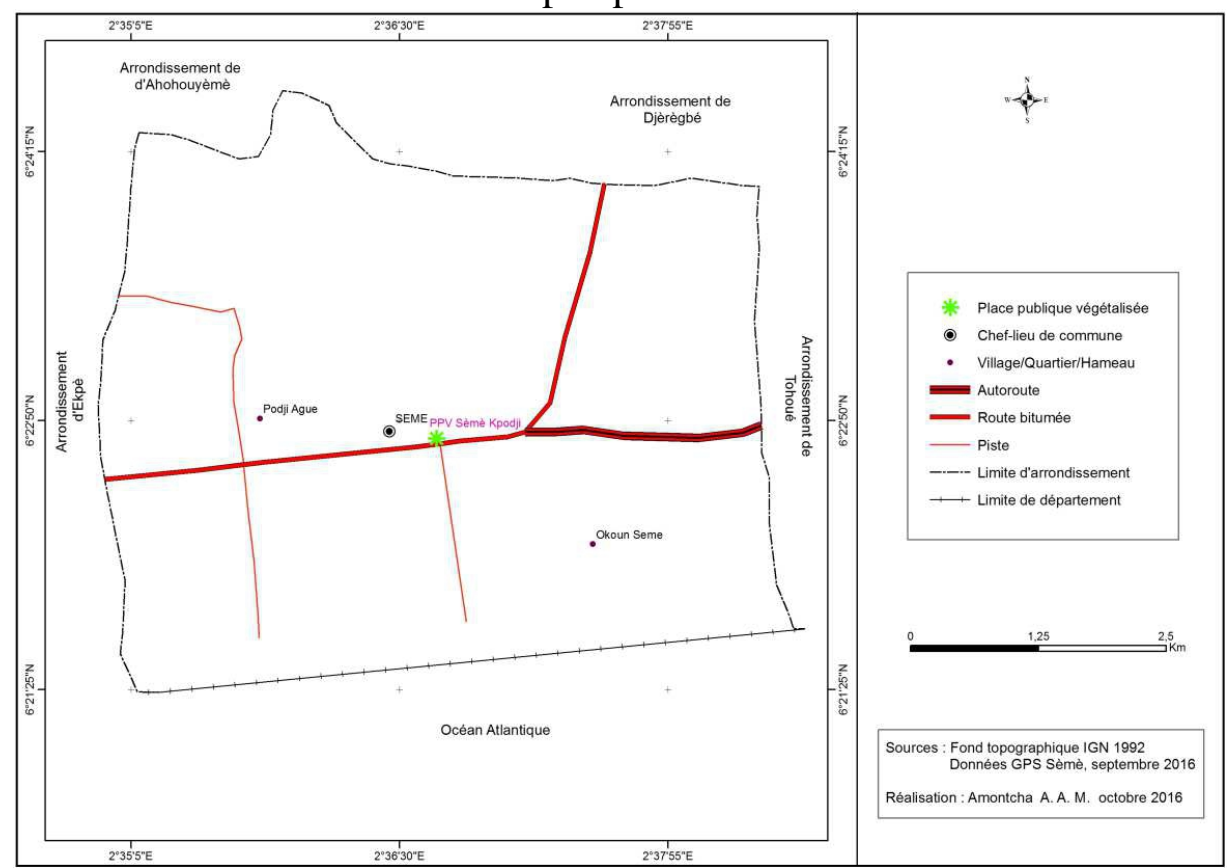

Figure 11 : Carte de répartition géographique des espaces verts publics de la ville de SèmèPodji 
Le seul espace vert public de la ville de Sèmè-Podji est localisé dans l'arrondissement central, c'est-à-dire celui de Sèmè-Podji (figure 11).

\section{Discussion}

Sur les douze types d'espaces verts reconnus par l'AIVF (Bouge, 2009), la présente recherche a révélé seulement quatre types d'espaces verts publics dans les villes du grand Nokoué avec quelques nuances entre les villes. Cette situation est due au fait que dans le cadre de cette recherche, c'est seulement les sites ouverts au public et à tout moment qui ont été considérés comme espaces verts publics.

La richesse en espaces verts publics des villes du grand Nokoué, 70 à Cotonou, 29 à Porto Novo, 8 à Ouidah, 6 à Abomey-Calavi et 1 à SèmèPodji est très faible voir insignifiant devant les 495 espaces verts de Châteauroux, 675 de Orléans et 655 de Tours (Richard, 2013). Cette différence s'explique par le fait que Richard (2013) a pris en compte, aussi bien les espaces verts privés que publics, ouverts ou fermé au publiques contrairement à ce qui a été fait dans cette étude. La différence peut aussi s'expliquer par le fait que les autorités en charge des villes de Châteauroux, Orléans et Tours réservent une bonne place aux espaces verts dans les plans de développement de leurs villes, font plus d'efforts de mobilisation de ressources et s'investissent mieux dans le suivi et l'entretien des espaces verts publics que les autorités des villes du grand Nokoué.

La présente recherche a révélé que la ville de Porto Novo dispose de 29 espaces verts publics. Cette quantité fait plus du double des 14 espaces verts publics dénombrés par Osseni (2014). Cette différence peut s'expliquer par le fait qu'Osseni (2014) dans son étude, n'a pas considéré les arbres d'alignement de voirie publics de Porto Novo comme des espaces verts publics.

Le ratio de $0,12 \mathrm{~m}^{2}$ d'espaces verts publics par habitant de la ville de Cotonou trouvé dans la présente recherche est inférieur au $0,18 \mathrm{~m}^{2}$ d'espaces verts publics par habitant trouvé par Houinsou (2009). Cette différence peut être dû au fait que certains espaces verts publics tels que le Jardin PTT, la place publique Aire Afrique, trouvé en 2009 par Houinsou n'existent plus aujourd'hui.

Ouidah est la ville du grand Nokoué qui a le ratio espace vert publics par habitant le plus élevé, à savoir $0,27 \mathrm{~m}^{2} \mathrm{~d}$ 'espace vert publics par habitant mais elle reste inférieur à la ville de Lomé (Togo) qui dispose de 0,75 $\mathrm{m}^{2}$ d'espace vert publics par habitant (Polorigni, 2014). Elle reste aussi très loin des $10 \mathrm{~m}^{2}$ d'espace vert publics par habitant recommandés par l'OMS (Bouge, 2009 ; Polorigni, 2014).

La faible richesse en espaces verts publics des villes du grand Nokoué peut aussi être due au manque d'organisation, d'anticipation et de 
planification de l'occupation du sol dans le grand Nokoué ; à l'insuffisance de moyens financier et à l'absence de stratégie de recherche de fonds nécessaire à l'aménagement de grand espaces verts. En effet, dans toutes les villes du grand Nokoué, les politiques d'aménagement sont a posteriori. Ce ne sont pas les municipalités (avec l'aide des architectes urbanistes) qui définissent les types d'aménagement à faire pour chaque type de milieux, mais les populations qui choisissent où s'installer, morcellent et occupent l'espace comme ils veulent. Quand enfin les autorités décident d'entreprendre des travaux de lotissement et de restructuration, les terres qu'elles parviennent à récupérer, pour l'aménagement des infrastructures sociocommunautaires, suite à l'application des coefficients de réduction sont peu. Conséquence, les espaces verts publics aménagés sont peu nombreux et peu large.

Selon les Chefs Services Techniques (CST) du grand Nokoué, les municipalités n'ont pas assez de moyens financiers pour aménager et gérer plus d'espaces verts, pour la simple raison que l'Etat centrale alloue peu/pas de fonds à ces aménagements. Les municipalités elles-mêmes n'entreprennent aucune démarche de mobilisation de ressources (rédaction de projets ambitieux, achat et réservation de grands domaines, développement de stratégie de mobilisation de fond) à cet effet. Pourtant ce n'est pas les institutions à vocation environnementales et sociales capables de financer l'aménagement des espaces verts qui manque, tant au niveau national, régional qu'international.

\section{Conclusion}

La présente recherche a montré que les espaces verts publics des villes du grand Nokoué appartiennent à quatre (4) types d'espaces verts : les espaces verts d'hommages (EVH), les arbres d'alignement de voirie publique (AAVP), les parcs et squares (PS) et les espaces verts d'accompagnement de voies (EVAV). Les espaces verts publics de type arbres d'alignement de voirie publique (AAVP) sont les plus dominant $(66,67 \%$ des espaces verts publics de la ville d'Abomey-Calavi, 62,85\% de Cotonou, 62,5\% de Ouidah et $48,28 \%$ de Porto-Novo). Les espaces verts publics sont inégalement répartis entre les villes du grand Nokoué. Cotonou est la ville la plus dense en espaces verts publics $\left(0,89\right.$ espace vert public $\left./ \mathrm{km}^{2}\right)$ et la plus couverte $(0,124 \%)$ en place publique végétalisée. Par contre la ville de Ouidah est la ville qui a le ratio espaces verts par habitant le plus élevé du grand Nokoué $\left(0,27 \mathrm{~m}^{2} / \mathrm{hbt}\right)$. Toutes les villes du grand Nokoué sont très loin des $10 \mathrm{~m}^{2}$ d'espace vert public recommandés par l'OMS pour chaque habitant. Vue la faible richesse en espaces verts publics des villes du grand Nokoué, il est nécessaire que les autorités municipales accordent plus d'intérêt et de places aux espaces verts publics (jardins, parcs, squares, etc.) dans les futurs plans 
de développement de leurs localités et fassent plus d'efforts de mobilisation de ressources pour leur aménagement et leur gestion durable.

\section{References:}

1. Akbari, H. (2002) : Shade trees reduce building energy use and CO2 emissions from power plants. Environmental Pollution, vol. 116, pp. 119-126

2. Beckett, KP, Freer-Smith, PH et Taylor, G. (2000) : Effective Tree Species for Local Air-Quality Management. Journal of Arboriculture, vol. 26, $\mathrm{n}^{\circ} 1$, pp. 12-19

3. Blalogoé, CP (2014) : Stratégies de lutte contre les inondations dans le grand Cotonou : diagnostic et alternative pour une gestion durable. Thèse de Doctorat Unique, Abomey-Calavi, Université d'Abomey-Calavi, 242 p.

4. Boko, M. (1988) : Climats et communautés rurales du Bénin : Rythmes climatiques et rythmes de développement. Thèse d'Etat eslettres, Dijon, Université de Bourgogne, 608 p.

5. Bouge, F. (2009): Caractérisation des espaces verts publics en fonction de leur place dans le gradient urbain - rural. Cas d'étude : la trame verte de l'Agglomération Tourangelle. Projet de Fin d'Etudes, Tours, Université François - Rabelais, 86 p.

6. Brack, CL (2002) : Pollution mitigation and carbon sequestration by an urban forest. Environmental Pollution, vol. 116, pp. 195-200

7. Breton, E., Bourges, F. et Draperi, C. (2015) : Approche globale et innovante de la conception technique et de la réalisation des couvertures: Focus sur la végétalisation. Agence Nationale de la Recherche - Programme Bâtiments et Villes Durables - CANOPEE - Tâche 5 - Cahier 7, 72 p.

8. Chen, WY et Jim, CY (2008): Assessment and Valuation of the Ecosystem Services Provided by Urban Forests. Ecosystem Services Provided by Urban Forests, vol. 5, pp. 53-83

9. Davies, ZG, Edmonson, JL, Heinemeyer, A., Leake, JR et Gaston, KJ (2011): Mapping an urban ecosystem service: quantifying aboveground carbon storage at a citywide scale. Journal of Applied Ecology, vol. 48, pp. 1125-1134

10. Dunnett, N. et Kingsbury, N. (2004) : Literally Green Facades. ArchitectureWeek. Mis en ligne le 28/07/2004, consulté le 13/03/2017

11. URL : http://www.architectureweek.com/2004/0728/environment_12.html

12. Gill, SE, Handley, JF, Ennos, AR et Pauleit, S. (2007) : Adapting cities for climate change: the role of the green infrastructure. Built 
Environment, vol. $33 \mathrm{n}^{\circ} 1$, pp. 115-133

13. Houinsou, TA (2009): Foresterie urbaine a Cotonou : diagnostic et approches de solution pour une gestion intégrée. Mémoire de DEA, Abomey-Calavi, Université d' Abomey-Calavi, 71 p.

14. INSAE (2013) : Résultats provisoires du RGPH 4. Cotonou, Bénin, MDAEP - Ministère du Développement, de l'Analyse Economique et de la Prospective, $8 \mathrm{p}$.

15. Janhäll, S. (2015): Review on urban vegetation and particle air pollution - Deposition and dispersion. Atmospheric Environment, vol. 105, pp. 130-137

16. En ligne, consulté le 05/04/2017

17. http://www.sciencedirect.com/science/article/pii/S135223101500075 $\underline{8}$

18. Jo, H. (2002) : Impacts of urban greenspace on offsetting carbon emissions for middle Korea. Journal of Environmental Management, vol. 64, pp. 115-126

19. Lamontagne, J. (2013): Protéger la végétation, non la détruire. Québec, Ministère de la Culture et des Communications, 28 p.

20. Nowak, DJ, Crane, DE et Stevens, JC (2006) : Air pollution removal by urban trees and shrubs in the United States. Urban Forestry \& Urban Greening, vol. 4, pp. 115-123

21. Osséni, AA, Tohozin, CAB, Toko Mouhamadou, I. et Sinsin, B. (2014) : Contribution des SIG dans l'analyse floristique des espaces verts dans la ville de Porto-Novo au Bénin. Revue Ivoirienne des Sciences et Technolie, vol. 23, pp. 103-121

22. Polorigni, B. (2014): Perceptions, tendances et préférences en foresterie urbaine: cas de la ville de Lomé au Togo. European Scientific Journal, vol. 10, n5, pp. 261-277

23. Richard, S. (2013) : La politique et la place des espaces verts en milieu urbain. Cas d'étude : les six préfectures départementales de la région Centre. Master 1 Géographie, Tours, Université François Rabelais, $119 \mathrm{p}$.

24. Texier, R. (1980) : Les ressources en eaux superficielles du Bénin, Cotonou, $345 \mathrm{p}$.

25. Volkoff, B. (1970): Carte pédologique de reconnaissance du Dahomey au 1/200000. Feuille de Porto-Novo, étude $n^{\circ} 121$. Cotonou-Bénin, OROSTOM, $80 \mathrm{p}+$ annexes.

26. Zhao, M., Kong, ZH, Escobedo, FJ et Gao, J. (2010) : Impacts of urban forests on offsetting carbon emissions from industrial energy use in Hangzhou, China. Journal of Environmental Management, vol. $91, n^{\circ} 4$, pp. 807-813 